\title{
A Fisioterapia Aquática no desenvolvimento motor na Artrogripose Múltipla Congênita: um relato de caso
}

\author{
Aquatic Physiotherapy in motor development in \\ Arthrogryposis Multiplex Congenita: a case report \\ Fisioterapia acuática en el desarrollo motor de la \\ Artrogriposis Múltiple Congénita: reporte de un caso
}

\author{
Luciana Alécio Cabanelas ${ }^{1}$, Douglas Martins Braga²
}

\begin{abstract}
1.Fisioterapeuta pela Faculdade do Clube Náutico Mogiano, Mestre em Ciências pela Universidade Federal de São Paulo, Fisioterapeuta do setor de Fisioterapia Aquática da Associação de Assistência à Criança Deficiente (AACD), Mogi das Cruzes-SP, Brasil.

2.Fisioterapeuta pela Universidade Cidade de São Paulo, Especialista em Neurologia e Neurociências, Mestre em Ciências da Saúde pela Universidade Federal de São Paulo, Supervisor em reabilitação do setor de Fisioterapia Aquática da Associação de Assistência à Criança Deficiente (AACD), São Paulo-SP, Brasil.
\end{abstract}

\begin{abstract}
Resumo
Introdução. A Artrogripose múltipla congênita (AMC) é uma doença rara, o prognóstico depende da severidade e do tratamento recebido. Objetivo. Descrever a reabilitação de uma criança com diagnóstico de AMC. Método. Relato de caso retrospectivo, descritivo dos atendimentos realizados no setor de fisioterapia aquática da Associação de Apoio à Criança Deficiente - Mogi das Cruzes. Resultados. Paciente do sexo feminino iniciou o tratamento no setor aos sete meses de idade e foi desenvolvido um programa de exercícios para a estimulação do desenvolvimento neuropsicomotor. Conclusão. A fisioterapia aquática pode oferecer um ambiente facilitador para crianças com AMC, favorecendo o aprendizado motor.

Unitermos. hidroterapia; amioplasia; desenvolvimento motor
\end{abstract}

\begin{abstract}
Introduction. Arthrogryposis Multiplex Congenita (AMC) is a rare disease, the prognosis depends on the severity and treatment received. Objective. To describe the rehabilitation of a child diagnosed with AMC. Method. Descriptive, retrospective case report of the consultations performed in the aquatic physiotherapy sector of the Associação de Apoio à Criança Deficiente - Mogi das Cruzes. Results. Female patient started treatment in the sector at seven months of age and an exercise program was developed to stimulate neuropsychomotor development. Conclusion: Aquatic physiotherapy can offer a facilitating environment for children with AMC, favoring motor learning.
\end{abstract}

Keywords. hydrotherapy; amyoplasia; motor development

\section{Resumen}

Introducción. La artrogriposis múltiple congénita (AMC) es una enfermedad rara, el pronóstico depende de la gravedad y el tratamiento recibido. Objetivo. Describir la rehabilitación de un niño diagnosticado de AMC. Método. Relato de caso retrospectivo de las consultas realizadas en el sector de fisioterapia acuática de la Associação de Assistência a Criança Deficiente - Mogi das Cruzes. Resultados. Paciente de sexo femenino, inició tratamiento en el sector a los siete meses de edad y se desarrolló un programa de ejercicios para estimular el desarrollo neuropsicomotor. Conclusión. La fisioterapia acuática puede ofrecer un entorno facilitador para los niños con AMC, favoreciendo el aprendizaje motor.

Palabras clave: hidroterapia; amioplasia; desarrollo motor 
Trabalho realizado na Associação de Assistência à Criança Deficiente (AACD), Mogi das Cruzes-SP, Brasil.

Conflito de interesse: não Recebido em: 08/04/2021 Aceito em: 26/10/2021

Endereço para correspondência: Luciana Alécio Cabanelas. Email: luciana.alecio@hotmail.com

\section{INTRODUÇÃO}

A Artrogripose múltipla congênita ( $A M C$ ) é uma condição rara, de etiologia indefinida, caracterizada por múltiplas contraturas presentes ao nascimento que tem como principal fator de risco a ausência de movimento fetal $^{1,2}$. O prognóstico depende da severidade do acometimento e o tratamento recebido ${ }^{3}$.

A reabilitação é desafiadora pois envolve um tratamento multidisciplinar e baseia-se na fisioterapia e um amplo espectro de técnicas cirúrgicas para correção de deformidades musculoesqueléticas ${ }^{4}$.

A fisioterapia aquática é definida como um programa de reabilitação que combina os conhecimentos do movimento humano e as propriedades físicas da água para melhorar as funções motoras ${ }^{5,6}$.

A flutuação facilita os movimentos antigravitacionais facilitando a movimentação de crianças que têm limitações na mobilidade e suas propriedades resistivas promovem 0 aumento de força muscular postural e das extremidades ${ }^{7,8}$.

A literatura é escassa sobre programas de reabilitação fisioterapêutica na $A M C^{9}$ e ainda mais restrita quanto a abordagem da fisioterapia aquática. Portanto o objetivo deste estudo foi descrever o processo de reabilitação no 
setor de fisioterapia aquática de uma criança com diagnóstico de AMC.

\section{MÉTODO}

Trata-se de um relato de caso retrospectivo, descritivo, com base na análise documental de prontuário dos atendimentos realizados no setor de fisioterapia aquática da Associação de Apoio à Criança DeficienteUnidade Mogi das Cruzes após aprovação no Comitê de Ética e Pesquisa sob 0 parecer CAAE: 36631320.3.0000.0085.

A amostra foi constituída por um paciente do sexo feminino com diagnóstico clínico de AMC atendida no setor de julho de 2012 a junho de 2016. Após aprovação do projeto, o responsável legal foi contactado via telefone, orientado e esclarecido quanto ao objetivo da pesquisa, e após concordar com a utilização dos dados do prontuário institucional, foi encaminhado o Termo de Consentimento Livre e Esclarecido (TCLE) e o Termo de Utilização de Imagem, ambos disponibilizados de maneira virtual, através de um endereço eletrônico.

Os dados coletados foram da avaliação e reavaliações qualitativas realizadas no período de atendimento, que abrangiam a aquisição ou aprimoramento dos marcos motores apresentados pela criança. Os dados estão descritos em ordem cronológica para o desenvolvimento da narrativa quanto os marcos do desenvolvimento motor e 
sua associação aos objetivos funcionais através da abordagem da Fisioterapia Aquática.

\section{RELATO DO CASO}

As alterações morfológicas foram detectadas no prénatal no quarto mês gestacional, com diminuição do líquido amniótico e presença de pé torto congênito. Ao nascimento apresentava adução e rotação interna de ombros, extensão de cotovelos e flexão de punho e dedos bilateralmente; quadril em rotação externa, joelhos em extensão e pés equino cavo varo parcialmente redutíveis. A paciente iniciou o tratamento na instituição aos dois meses de idade com o uso do gesso seriado e realização de duas tenotomias percutâneas do tendão do calcâneo, finalizando a intervenção aos seis meses de idade. Concomitante, realizava o uso noturno da órtese de Dennis Brown.

Iniciou o tratamento no setor de fisioterapia aquática aos sete meses de idade em piscina coberta e aquecida com temperatura média da água de $32^{\circ} \mathrm{C}$. As sessões foram realizadas uma vez por semana, com duração de 35 minutos e o programa de exercícios incluíam alongamentos, mobilização das articulações e estimulação do desenvolvimento neuropsicomotor de acordo com os objetivos funcionais delineados.

\section{RESULTADOS}

$\mathrm{Na}$ avaliação inicial em supino a paciente apresentava movimentação ativa de flexão lateral e anterior de tronco, 
flexão de quadril com joelhos em extensão. Realizava o rolar até decúbito lateral e em prono com auxílio, apresentava boa extensão de tronco e cervical. Colocada sentada o tronco se mantinha instável e precisava de apoio para se manter na posição. Assim, dos 07-13 meses o objetivo funcional foi favorecer o rolar e a manutenção da postura sentada para a interação e exploração do ambiente.

Com aquisição da sedestação sem apoio, dos 13 aos 16 meses o objetivo funcional foi o deslocamento sentado e as reações de proteção. Visto a inabilidade destas reações ocorrerem com os membros superiores devido as contraturas articulares, este tipo de treinamento foi enfatizado para favorecer a consciência corporal e o ajuste de tronco. Aos 16 meses a paciente apresentava boa evolução do desempenho da função com melhora da agilidade e mudanças de direção, sendo a principal forma de locomoção no período. A aquisição e aprimoramento do ortostatismo foi enfatizado dos 16 aos 31 meses, com foco nas adaptações para a passagem do sentado para de pé e equilíbrio estático e dinâmico. Com a melhora do desempenho na postura, o treino de marcha foi iniciado e aprimorado até o momento da alta, onde a criança apresentava marcha comunitária independente.

No Quadro 1 estão descritos os manuseios realizados para a estimulação dos objetivos funcionais propostos nos diferentes períodos do desenvolvimento motor. 
Quadro 1. Descrição dos objetivos e manuseios ao longo do tratamento.

\begin{tabular}{|c|c|c|}
\hline IDADE & OBJETIVO & MANUSEIOS \\
\hline \multirow{5}{*}{$\begin{array}{l}07-13 \\
\text { meses }\end{array}$} & \multirow[b]{2}{*}{ ROLAR } & $\begin{array}{l}\text { Estimulou-se a rotação no eixo axial através da flutuação } \\
\text { horizontal com a turbulência e o efeito metacêntrico. }\end{array}$ \\
\hline & & $\begin{array}{l}\text { Em flutuação foi estimulada a abdução de ombro, o alcance e o } \\
\text { acompanhamento visual para favorecer o rolar. }\end{array}$ \\
\hline & \multirow{3}{*}{ SENTAR } & $\begin{array}{l}\text { Utilizando a rotação transversal com suporte em quadril foi } \\
\text { estimulada a flexão de tronco para a passagem para a posição } \\
\text { sentada. }\end{array}$ \\
\hline & & $\begin{array}{l}\text { Estimulado } \circ \text { ajuste postural de tronco com deslocamentos } \\
\text { laterais e antero-posterior em diferentes níveis de imersão. }\end{array}$ \\
\hline & & $\begin{array}{l}\text { Mobilidade de tronco e membros superiores: estimulando o } \\
\text { alcance de brinquedos na superfície da água com flutuadores } \\
\text { posicionados em região proximal de membros superiores } \\
\text { favorecendo a amplitude de ombro. }\end{array}$ \\
\hline $\begin{array}{l}13-16 \\
\text { meses }\end{array}$ & $\begin{array}{l}\text { Deslocamento } \\
\text { sentado }\end{array}$ & $\begin{array}{l}\text { Com nível de imersão em região axilar e em declive para } \\
\text { facilitação do movimento e da ativação muscular e conforme } \\
\text { evolução foi diminuído o nível de imersão gradativamente e a } \\
\text { inclinação, de modo a oferecer resistência e dificuldade. }\end{array}$ \\
\hline $\begin{array}{l}16-25 \\
\text { meses }\end{array}$ & Ortostatismo & $\begin{array}{l}\text { Realizado em diferentes níveis de imersão, com suporte do } \\
\text { terapeuta em quadril e uso de caneleiras de peso em tornozelos } \\
\text { para melhor manutenção da postura. }\end{array}$ \\
\hline $\begin{array}{l}25-31 \\
\text { meses }\end{array}$ & $\begin{array}{l}\text { Estratégias de } \\
\text { equilíbrio }\end{array}$ & $\begin{array}{l}\text { Na posição ortostática foi incentivada a movimentação ativa de } \\
\text { tronco e membros superiores apoiados em tapete de EVA na } \\
\text { superfície da água. }\end{array}$ \\
\hline $31-54$ & Marcha & $\begin{array}{l}\text { Realizado em diferentes níveis de imersão, aclive, declive, com } \\
\text { utilização de caneleiras de peso em tornozelos para estabilização } \\
\text { dos pés no fundo da piscina ou flutuadores para facilitar a } \\
\text { mobilidade de quadril a favor do empuxo para a fase de balanço. } \\
\text { Treino para transpor obstáculos, subir e descer degrau. }\end{array}$ \\
\hline
\end{tabular}

\section{DISCUSSÃO}

As contraturas presentes ao nascimento na $A M C$ afetam o desenvolvimento motor, e o treino das trocas posturais deve ser adaptado para que a criança possa vivenciar as habilidades motoras mais próximas do desenvolvimento normal. Neste sentido, a água cria um ambiente adaptável ao exercício, pois fornece suporte por meio de flutuabilidade, e a capacidade de ativar músculos que têm dificuldade em superar as forças gravitacionais são 
as principais razões pelas quais os exercícios em imersão são apropriados aos indivíduos com restrições motoras ${ }^{10}$.

Sabe-se que as crianças com AMC o tronco se apresenta com força normal e apesar da sensibilidade não sofrer alterações, há a diminuição dos reflexos nos membros ${ }^{11}$. A abordagem através do conceito do Halliwick ${ }^{12}$ para trabalhar o controle das rotações, favorece o estímulo do controle corporal em um ambiente tridimensional, para a aquisição do controle de tronco e ações musculares reativas de membros, com ênfase no ajuste postural e endireitamento.

As crianças com AMC atingem níveis funcionais diferentes dependendo do acometimento articular $e$ muscular e o arrastar na postura sentada é uma forma de deslocamento desenvolvida para serem funcionais ${ }^{13}$. Durante estudo utilizando a intervenção da fisioterapia aquática no deslocamento de uma criança com AMC, os autores observaram melhora do controle de tronco $\mathrm{e}$ estabilidade na postura sentada assim como melhora da velocidade do arrastar nesta posição ${ }^{13}$. Estes dados corroboram com o atual estudo onde o estímulo do deslocamento sentado em diferentes níveis de imersão favoreceu essa forma de deslocamento domiciliar.

A ativação muscular de quadríceps e músculos pélvicos tendem a ser preservados nestes pacientes ${ }^{14}$, 0 que favorece a manutenção e exploração da postura ortostática em um meio facilitador como a piscina. Além da interação entre a informação sensorial e o desempenho 
motor, o controle postural também depende da maturação das estruturas envolvidas, e das experiências motoras ${ }^{15}$, já que as estratégias posturais devem se adaptar aos diferentes estímulos e ambientes. A imersão é uma forma de perturbação sensorial e mecânica e acredita-se que pode melhorar a estabilidade postural em solo ${ }^{16}$.

A pressão hidrostática e a viscosidade oferecem feedback sensorial e proprioceptivo e influencia o sistema de controle postural e na manutenção do equilíbrio ${ }^{17}$. Assim, neste estudo a aquisição do ortostatismo, marcha e a estratégias de equilíbrio puderam ser estimulados de forma segura, o que favoreceu o aprendizado motor.

Os pacientes com AMC com prognóstico de marcha, iniciam a deambulação comunitária com suporte antes dos dois anos e meio de idade em conjunto com a maturação esquelética, e de forma independentemente aos nove anos de idade ${ }^{18}$, e esses dados corroboram com os achados neste estudo onde a marcha comunitária foi alcançada e realizada de forma independente no momento da alta aos 4 anos e 5 meses de idade.

\section{CONCLUSÃO}

A fisioterapia aquática pode oferecer um ambiente facilitador para crianças com AMC, com maiores possibilidades de movimentação ativa, favorecendo o aprendizado motor e aquisição posturais e habilidades que podem ser transferidas para o solo. 


\section{REFERÊNCIAS}

1.Jones T, Miller R, Street JT, Sawatzky B. Validation of the Oswestry Disability Index for pain and disability in arthrogryposis multiplex congenita. Ann Phys Rehabil Med 2019;62:92-7. http://dx.doi.org/10.1016/j.rehab.2018.05.1319

2.Sucuoglu H, Ornek NI, Caglar C. Arthrogryposis Multiplex Congenita: Multiple Congenital Joint Contractures. Case Rep Med 2015;2015:379730. http://dx.doi.org/10.1155/2015/379730

3.Shinde A, Mhaske SN, Bhate SN. Arthrogryposis Multiplex Congenita: A Rare Case Report. VIMS Health Sci J 2020;7:85-6. https://doi.org/10.46858/vimshsj.7305

4. Kowalczyk B, Feluś J. Arthrogryposis: an update on clinical aspects, etiology, and treatment strategies. Arch Med Sci 2016;12:10-24. http://dx.doi.org/10.5114/aoms.2016.57578

5.Güeita-Rodríguez J, García-Muro F, Rodríguez-Fernández ÁL, CanoDíez B, Chávez-Santacruz D, Palacios-Ceña D. Preliminary Aquatic Physical Therapy Core Sets for Children and Youth With Neurological Disorders: A Consensus Process. Pediatr Phys Ther 2019;31:272-8. http://dx.doi.org/10.1097/PEP.0000000000000624

6.Terrens AF, Soh SE, Morgan PE. The efficacy and feasibility of aquatic physiotherapy for people with Parkinson's disease: a systematic review. Disabil Rehabil 2018;40:2847-56. http://dx.doi.org/10.1080/09638288.2017.1362710

7.McManus BM, Kotelchuck M. The effect of aquatic therapy on functional mobility of infants and toddlers in early intervention. Pediatr Phys Ther 2007;19:275-82. http://dx.doi.org/10.1097/PEP.0b013e3181575190

8. Roostaei M, Baharlouei $H$, Azadi $H$, Fragala-Pinkham MA. Effects of Aquatic Intervention on Gross Motor Skills in Children with Cerebral Palsy: A Systematic Review. Phys Occup Ther Pediatr 2017;37:496515. http://dx.doi.org/10.1080/01942638.2016.1247938

9.Binkiewicz-Glinska A, Sobierajska-Rek A, Bakula S, Wierzba J, Drewek K, Kowalski IM, et al. Arthrogryposis in infancy, multidisciplinary approach: case report. BMC Pediatr 2013;13:184. http://dx.doi.org/10.1186/1471-2431-13-184

10.Ballington SJ, Naidoo R. The carry-over effect of an aquatic-based intervention in children with cerebral palsy. Afr J Disabil 2018;7:361. http://dx.doi.org/10.4102/ajod.v7i0.361

11. Hall JG, Aldinger KA, Tanaka KI. Amyoplasia revisited. Am J Med Genet 2014;164:700-30. http://dx.doi.org/10.1002/ajmg.a.36395 12.Gurpinar B, Kara B, Idiman E. Effects of aquatic exercises on postural control and hand function in Multiple Sclerosis: Halliwick versus Aquatic Plyometric Exercises: a randomised trial. J Musculoskelet Neuronal Interact 2020;20:249-55. https://www.ncbi.nlm.nih.gov/pmc/articles/PMC7288381/pdf/JMNI20-249.pdf

13. Nicolini-Panisson RDA, Hengles RC, Mattos DCG. Atuação da fisioterapia aquática funcional no deslocamento na postura sentada 
na amioplasia congênita: estudo de caso. Sci Med 2015;24:399-403. http://dx.doi.org/10.15448/1980-6108.2014.4.17752

14.Donohoe M, Pruszcynski B, Rogers K, Bowen JR. Predicting Ambulatory Function Based on Infantile Lower Extremity Posture Types in Amyoplasia Arthrogryposis. J Pediatr Orthop 2019;39:e5315. http://dx.doi.org/10.1097/BPO.0000000000001322

15.Sá CDSC, Boffino CC, Ramos RT, Tanaka C. Development of postural control and maturation of sensory systems in children of different ages a cross-sectional study. Braz J Phys Ther 2018;22:706. http://dx.doi.org/10.1016/j.bjpt.2017.10.006

16. Marinho-Buzelli AR, Rouhani H, Masani K, Verrier MC, Popovic MR. The influence of the aquatic environment on the control of postural sway. Gait Posture 2017;51:70-6. http://dx.doi.org/10.1016/j.gaitpost.2016.09.009

17.Saleh MSM, Rehab NI, Aly SMA. Effect of aquatic versus land motor dual task training on balance and gait of patients with chronic stroke: A randomized controlled trial. NeuroRehabilitation 2019;44:485-92. http://dx.doi.org/10.3233/NRE-182636

18. Fassier A, Wicart P, Dubousset J, Seringe R. Arthrogryposis multiplex congenita. Long-term follow-up from birth until skeletal maturity. J Child Orthop 2009;3:383-90. http://dx.doi.org/10.1007/s11832-009-0187-4 